\title{
Physical insight into a Mach 7.2 compression corner flow
}

\author{
Konstantinos Ritos*, Ioannis W. Kokkinakis ${ }^{\dagger}$ and Dimitris Drikakis ${ }^{\ddagger}$ \\ University of Strathclyde, Glasgow, G1 1XJ, UK
}

\begin{abstract}
High-order implicit Large Eddy Simulations were conducted to study shock-boundary layer interaction around a $33^{\circ}$ compression corner at Mach 7.2 and Reynolds number of $R e_{\theta}=3,500$ based on the momentum thickness. A grid-convergence study was performed to reduce the computational uncertainty and the results were compared with experiments and theoretical predictions. Furthermore, the turbulent flow properties were analysed with respect to the Reynolds normal stress, skewness and flatness, and conclusions were drawn regarding the shock boundary layer interaction behavior.
\end{abstract}

\section{Nomenclature}

$\alpha \quad$ Ramp angle, ${ }^{\circ}$

$\beta \quad$ Inviscid shock angle, ${ }^{\circ}$

$\Delta y \quad$ Grid spacing, $\mathrm{m}$

$\Delta y^{+}$Grid spacing scaled with inner variables

$\delta \quad$ Boundary layer thickness, mm

$\nu \quad$ Kinematic viscosity, $\mathrm{m}^{2} / \mathrm{s}$

$\rho \quad$ Density, $\mathrm{kg} / \mathrm{m}^{3}$

$\tau_{u u} \quad$ Normalized Reynolds normal stress

$C_{f} \quad$ Skin friction coefficient

$C_{p} \quad$ Pressure coefficient

$F_{u} \quad$ Flatness

$k$ Wavenumber, radians $/ \mathrm{m}$

$M \quad$ Mach number

$N \quad$ Number of grid points

$P \quad$ Pressure, $\mathrm{Pa}$

Pr Prandtl number

$R e_{\delta} \quad$ Boundary layer thickness Reynolds number

$R e_{\theta} \quad$ Momentum thickness Reynolds number

$R e_{\tau}$ Friction velocity Reynolds number

$S_{u} \quad$ Skewness

St Stanton number

$T$ Temperature, $\mathrm{K}$

$u \quad$ Stream-wise velocity, $\mathrm{m} / \mathrm{s}$

$u^{\prime} \quad$ Velocity fluctuation, $\mathrm{m} / \mathrm{s}$

$u_{\tau}$ Friction velocity, $\mathrm{m} / \mathrm{s}$

Subscript

$\infty \quad$ Free-stream

$\zeta \quad$ Parallel to the ramp direction

$e \quad$ Boundary layer edge

\footnotetext{
*Lecturer, University of Strathclyde, Glasgow, UK, AIAA Member.

${ }^{\dagger}$ Lecturer, University of Strathclyde, Glasgow, UK.

${ }^{\ddagger}$ Professor of Engineering Science \& Executive Dean of the Faculty of Engineering at the University of Strathclyde, Glasgow, UK, Senior Life Member AIAA.
} 
$p \quad$ Value on specific position

$w \quad$ Wall

$x \quad$ Stream-wise direction

$y, \eta \quad$ Wall normal direction

$z \quad$ Span-wise direction

\section{Introduction}

High resolution simulations of hypersonic shock wave turbulent boundary layer interaction (SWBLI) have significantly enhanced our understanding of these flows, particularly in the areas where turbulence measurements at high Mach numbers are difficult to be performed. ${ }^{1}$ Past numerical ${ }^{2,3}$ and experimental studies $^{4-7}$ of hypersonic SWBLI have shown that the turbulent stresses are amplified. Görtler-like vortices have been also observed at Mach 7 around a compression ramp and it was suggested that these vortices are responsible for the low-frequency motion of the shock wave. ${ }^{3}$

The aim of this study is to provide further insight into the hypersonic SWBLI by performing implicit Large Eddy Simulations (iLES) of a Mach 7.2 turbulent boundary layer over a $33^{\circ}$ compression ramp. The ramp angle and free-stream properties have been chosen according to the experimental set up of Schreyer et al. ${ }^{7}$ It is expected that one or more shocks will form in the supersonic and hypersonic parts of the flow. The flowfield is affected by the interaction between this shock system and the incoming boundary layer. In this interaction region large gradients of pressure and skin friction will occur. The high ramp angle will create a significantly large pressure rise which will cause the flow to separate (separation bubble). The SWBLI is inherently unsteady and the shock fluctuates with a relatively low frequency around its mean position in the stream-wise direction. Reynolds normal stresses and high-order statistics are used to highlight flow features and to identify the turbulence structure around the compression corner.

The iLES approach and the computational setup are presented in Section II. A grid convergence study and validation of the simulation results against experiments are provided in Section III. The high-order statistics and turbulence topology are discussed in Section IV and the main conclusions drawn from this study are presented in Section V.

\section{Computational Model}

iLES are used in the framework of the high-order code CNS3D that has been successfully applied to a range of turbulent studies, including supersonic and hypersonic transitional and turbulent flows. ${ }^{8-10}$ CSN3D employs block-structured grids and solves the full Navier-Stokes equations using a finite volume Godunovtype method for the convective terms. The "Harten, Lax, van Leer, and (the missing) Contact" (HLLC) Riemann solver ${ }^{11}$ in conjunction with a ninth-order Weighted-Essentially-Non-Oscillatory (WENO) scheme ${ }^{12}$ are used for the advective terms. The viscous terms are discretized by a second-order central scheme and a third-order accurate Runge-Kutta method ${ }^{13}$ is employed for the time integration.

The flow case considered here is a hypersonic flow over a $\alpha=33^{\circ}$ compression ramp followed by an expansion corner with the same deflection angle (Figure 1). The incoming flow corresponds to a turbulent boundary layer at Mach 7.2 with a thickness $\delta=5 \mathrm{~mm}$. Based on the free-stream properties (Table 1 ) and the reference length $\delta$, the incoming flow has a Reynolds number of $R e_{\delta}=102,731$. Periodic boundary conditions are implemented in the span-wise $(z)$ direction. In the wall-normal $(y)$ direction, a no-slip isothermal wall (with a temperature $\mathrm{T}_{w}$ of $340 \mathrm{~K}$ ) is used. ${ }^{14}$ High-order implementation of the boundary conditions requires fictitious cells to be added inside the wall. The velocity components on these cells are linearly extrapolated from the computational cells inside the domain. The temperature is also linearly extrapolated using the specified wall temperature. The density is calculated from the equation of state considering zero pressure gradient normal to the wall. Supersonic outflow conditions are applied to the outlet and far-field conditions are applied to the upper boundary.

Table 1: Simulation parameters

\begin{tabular}{cccccccccc}
$\delta(\mathrm{mm})$ & $U_{\infty}(\mathrm{m} / \mathrm{s})$ & $P_{\infty}(\mathrm{Pa})$ & $T_{\infty}(\mathrm{K})$ & $M_{\infty}$ & $\rho_{\infty}\left(\mathrm{kg} / \mathrm{m}^{3}\right)$ & $T_{w}(\mathrm{~K})$ & $R e_{\delta}$ & $R e_{\theta}$ & $R e_{\tau}$ \\
\hline 5.0 & 1,146 & $1,365.6$ & 63.06 & 7.2 & 0.0755 & 340 & 102,731 & 3,500 & 198
\end{tabular}




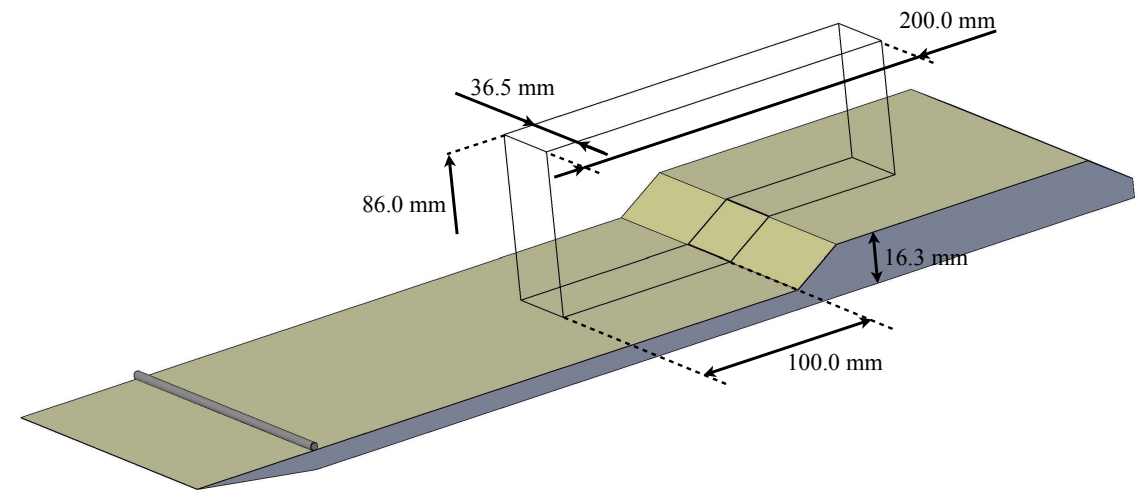

Figure 1: Schematic representation of the simulation domain with dimensions in mm.

A synthetic turbulent inflow boundary condition based upon the digital filter (DF) method documented in previous publications ${ }^{15-17}$ and validated in the framework of the present iLES code CNS3D ${ }^{9}$ is used to produce the turbulent boundary layer. According to the DF method, instead of using a white-noise random perturbation at the inlet, energy modes within the Kolmogorov inertial range scaling with $k^{-5 / 3}$, where $k$ is the wavenumber, are introduced into the turbulent boundary layer. No large-scale energy modes, scaling with $k^{4}$, are introduced. The mean turbulent profile is obtained from the experimental study of Schreyer, ${ }^{6}$ whereas the integral length scales chosen in this study are $0.4 \delta$ for the stream-wise direction and $0.5 \delta$ for the span-wise and wall normal directions, respectively.

Following typical resolution recommendations for LES and DNS simulations ${ }^{18-20}$ three relative fine grids were used in this study ranging from fine wall-resolved LES (G1) to under-resolved DNS (G3). The grid is clustered near the corner in the stream-wise direction and near the wall in the wall-normal direction. The number of grid points and the grid spacing are summarized in Table 2 along with the LES and DNS recommendations from the literature. The present grid spacing $(\Delta y)$ is scaled using the conventional inner variable method $\Delta y^{+}=u_{\tau} \Delta y / \nu_{w}$, where $u_{\tau}=\sqrt{\tau_{w} / \rho_{w}}$ is the friction velocity, $\nu_{w}$ is the near wall kinematic viscosity, $\tau_{w}$ is the near wall shear stress, and $\rho_{w}$ is the near wall density.

Table 2: Grid parameters

\begin{tabular}{ccccccccc} 
& $N_{x}$ & $N_{y}$ & $N_{z}$ & $\Delta x_{\min }^{+}$ & $\underline{\Delta x_{\max }^{+}}$ & $\Delta y_{w}^{+}$ & $\Delta y_{e}^{+}$ & $\Delta z^{+}$ \\
\hline G1 & 401 & 167 & 107 & 11.48 & $\underline{41.14}$ & 1.0 & 7.37 & 13.48 \\
G2 & 601 & 249 & 161 & 7.7 & 27.35 & 1.0 & 4.26 & 8.96 \\
G3 & 801 & 333 & 213 & 5.77 & 20.4 & 0.5 & 3.65 & 6.77 \\
LES $^{18-20}$ & - & - & - & 50 & 150 & $\leq 1.0$ & - & $15-40$ \\
DNS $^{18-20}$ & - & - & - & 10 & 20 & $<1.0$ & - & $5-10$
\end{tabular}

The structure of turbulence at the compression/expansion ramp is clearly visualized by iso-surfaces of the compressible Q-criterion ${ }^{21}$ for grid G3 in Figure 2. The iso-surfaces are colored with the stream-wise velocity clearly showing the flow separation at the corner. The density gradient is also plotted in greyscale indicating the position and the thickness of the shock. In the following section, the grid convergence for the three grids will be shown along with the mean flow properties. The flow statistics are computed by averaging in time over at least seven flow-throughs and spatially in the span-wise direction. The total simulation time for each case is equal to at least twelve flow-throughs, with the first five omitted from the calculations for statistical purposes. The statistical convergence of the simulations has been tested by comparing the results between fifteen and seven flow-throughs with grid G1 resulting in less than $2 \%$ difference. 


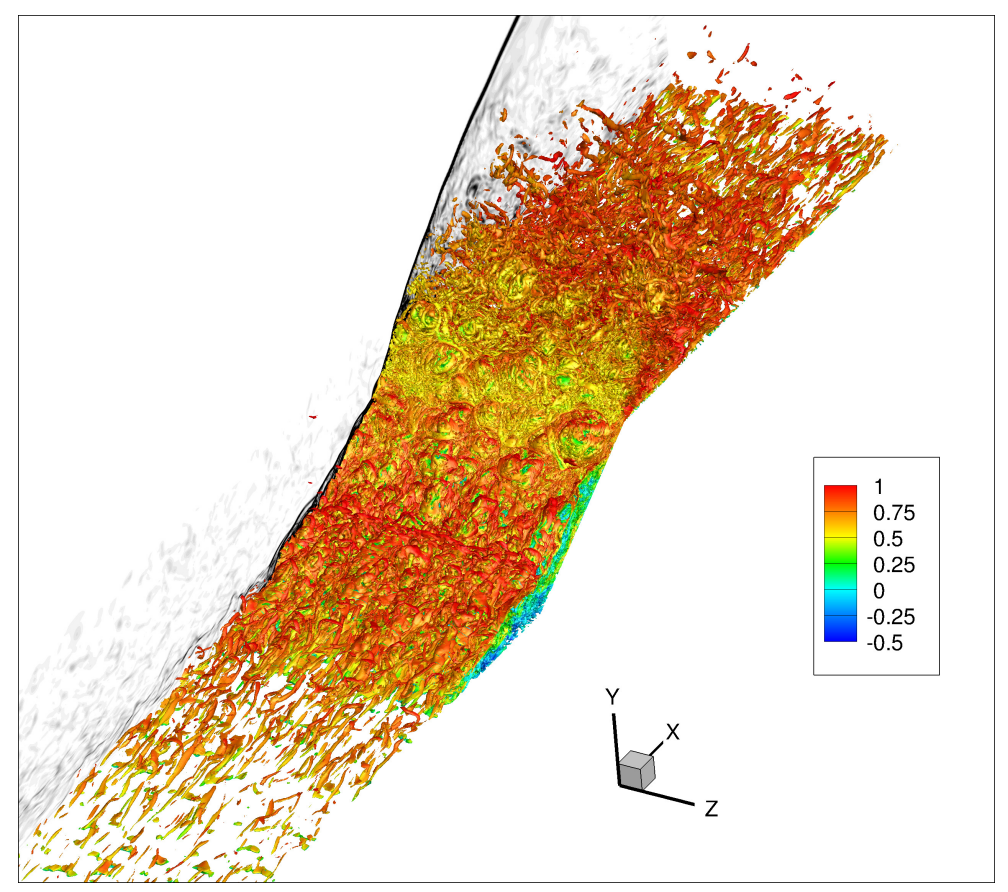

Figure 2: Iso-surfaces of compressible Q-criterion colored by stream-wise velocity indicating the flow separation at the corner. The density gradient is also plotted in grayscale on the side of the plot highlighting the position and the thickness of the shock.

\section{Grid convergence and iLES accuracy}

A grid convergence study is performed using the three grids listed in Table 2 in order to justify the use of G3 in the further analysis. In Figure 3 the skin friction coefficient $\left(C_{f}\right)$, the pressure coefficient $\left(C_{p}\right)$ and the Stanton number $(S t)$ are plotted along the stream-wise direction. The $C_{f}$ indicates the size of the separation bubble with its onset being the point where $C_{f}$ becomes negative. The two finer grids, G2 and G3, predict a significantly smaller bubble compared to grid G1. The largest $C_{f}$ value is obtained after the expansion corner.

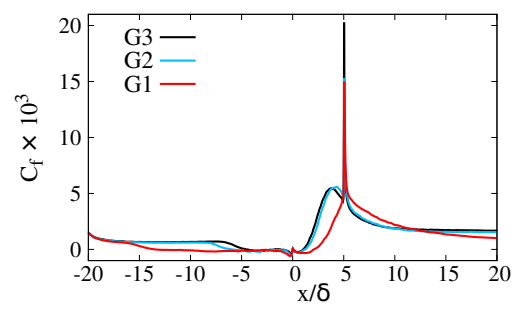

(a)

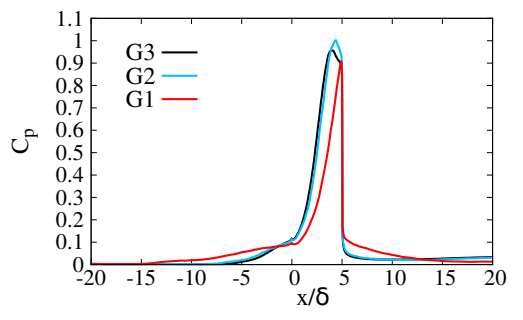

(b)

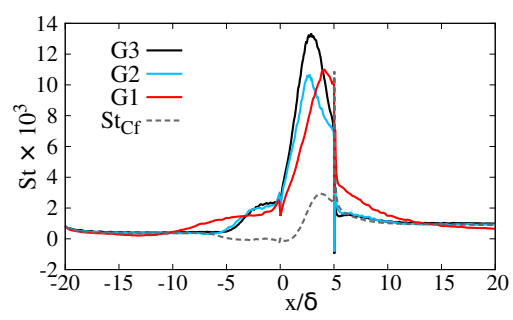

(c)

Figure 3: Highlighting grid converge through the comparison of the (a) skin friction coefficient, (b) pressure coefficient and (c) Stanton number along the stream-wise direction of the simulation domain. The analogy between the skin friction coefficient and the Stanton number is also plotted in (c) with $S t_{C f}=0.5 * C_{f} / \operatorname{Pr}^{0.67}$.

The maximum value of $C_{p}$ is observed at the re-attachment point and sharply decreases after the expansion corner (Figure 3b). The Stanton number relates the heat transfer into the fluid with the thermal capacity of the fluid, and it can be related to the skin friction coefficient through the relation $S t=0.5 * C_{f} / \operatorname{Pr}^{0.67}$. This analogy holds for turbulent or laminar flows with small thermal and velocity gradients close to the wall, failing to match the calculations in the region $-5<x / \delta<5$ (Figure 3c). In the region of the separation bubble and the ramp, significant thermal and velocity gradients occur under the present flow conditions. The two finer grids show excellent agreement with each other in all the shown predictions, whereas deviations 
occur at the coarsest grid.

The accuracy of iLES is assessed through comparison with available experimental measurements ${ }^{7}$ of mean velocity and velocity fluctuations at various positions (Table 3 ) along the compression/expansion ramp. All profiles are plotted normal to the local wall direction.

Table 3: Positions of comparison of ilES and experiment.

\begin{tabular}{cccc} 
& position & $x / \delta$ & $\zeta / \delta$ \\
\hline Upstream of the ramp corner & 1 & -4 & \\
& 2 & -3 & \\
3 & -2 & \\
& 4 & -1 & \\
\hline At the ramp corner & 5 & 0 & \\
\hline On the ramp & 6 & & 1 \\
& 7 & & 2 \\
& 8 & & 3 \\
At the expansion corner & 9 & & 4 \\
& 10 & & 5 \\
\hline Downstream of the expansion corner & 11 & & 6 \\
\hline & 12 & 6 & \\
& 13 & 7 & \\
& 14 & 8 & \\
& 15 & 9 & \\
& 16 & 10 &
\end{tabular}

Figure 4 shows velocity profiles parallel to the wall for all the comparison positions. The agreement is good upstream of the ramp corner and on the ramp corner. The negative mean stream-wise velocities in the recirculation region are predicted by the iLES calculations but are not shown in the experimental PIV data. However, Schreyer et al. ${ }^{7}$ mention in their paper that a separation region was visually observed in flow visualizations and that the deficiency in the measurements is attributed to the lack of PIV resolution in the near-wall region. This can also be responsible for the discrepancies between iLES and experiments near the wall on the ramp and around the expansion corner. The first velocity profile in Figure 4b (position 6 in Table 3) shows a sudden jump close to the boundary layer edge, which is due to the shock. Further downstream of the expansion corner the results show better agreement apart from the near-wall region.

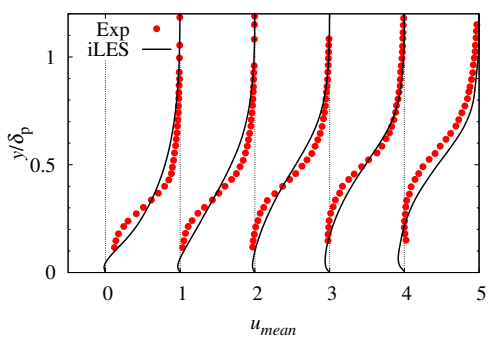

(a)

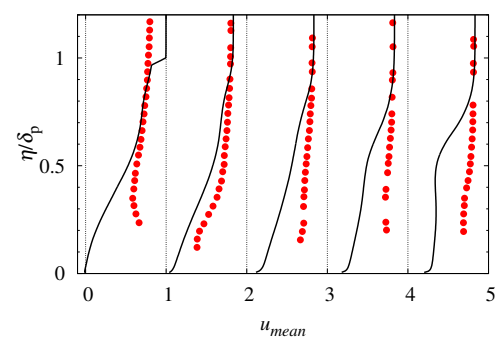

(b)

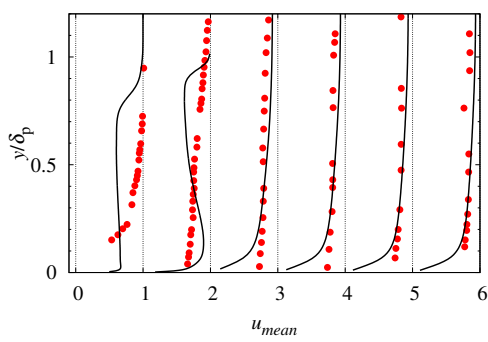

(c)

Figure 4: $u_{\text {mean }}$ profiles for various positions along the simulation domain. The normal distance from the wall has become dimensionless using the local boundary layer thickness $\delta_{p}$ at each position. Experimental data is from the publication of Schreyer et al. ${ }^{7}$ (a) Positions upstream of the ramp corner; (b) on the ramp; and (c) downstream of the expansion corner. The legend in Figure (a) applies to all subfigures.

The velocity fluctuations $u^{\prime}$ are shown in Figure 5c. iLES and experiments show good agreement around the ramp corner and downstream of the expansion corner. Near wall discrepancies, which are more pro- 
nounced on the ramp and near the expansion corner, are attributed to the deficiencies of the experimental approach, as discussed above. Wall normal velocities and velocity fluctuations are not compared with the experimental data as those are considered preliminary due to inadequate particle displacement between successive PIV images. ${ }^{7}$ Further experimental studies using different measurement techniques are necessary in order to obtain further confidence in the results.

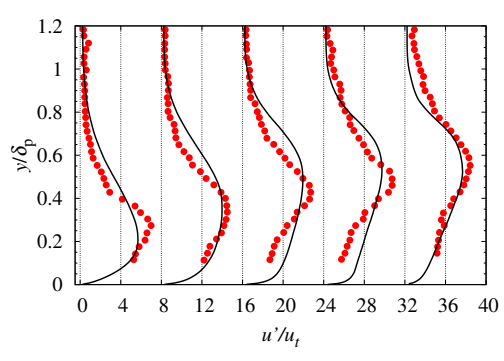

(a)

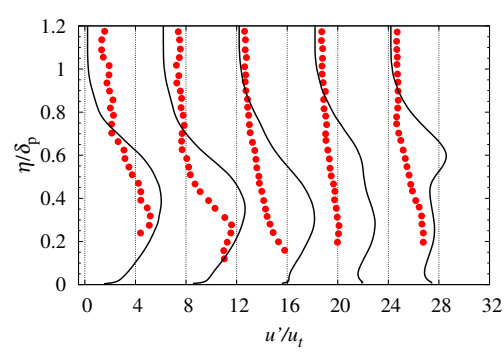

(b)

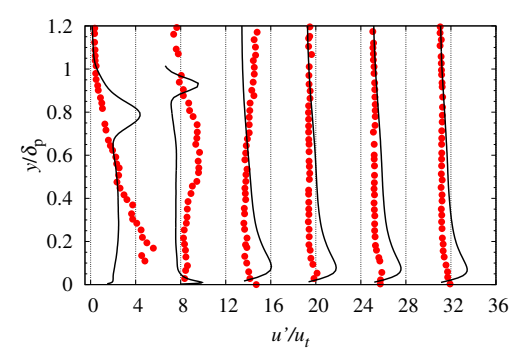

(c)

Figure 5: Velocity fluctuation $\left(u^{\prime}\right)$ profiles normalized by the experimental friction velocity $\left(u_{\tau}=57.3\right.$ $\mathrm{m} / \mathrm{s})^{7}$ for various positions along the simulation domain. The normal distance from the wall has become dimensionless using the local boundary layer thickness $\delta_{p}$ at each position. Experimental data is from the publication of Schreyer et al. ${ }^{7}$ (a) Positions upstream of the ramp corner; (b) on the ramp; and (c) downstream of the expansion corner. The legend in Figure 4a applies to all subfigures here.

The iLES results are also compared with the inviscid shock theoretical predictions (Figures 6 and 7). For the present flow conditions the inviscid theory predicts a shock angle $\beta=43.9^{\circ}$. The theoretically predicted inviscid shock is indicated by a red dashed line in Figure 6, where the numerical Schlieren $(N S=$ $0.8 * e^{-0.1|\nabla \rho|}$ ) (averaged in time) in the span-wise direction is shown. The angle of the numerically predicted shock downstream of the separation region agrees with the inviscid solution. The mean profiles of pressure, total pressure, Mach number and temperature calculated on the ramp before the expansion corner show that the post-shock flow outside of the boundary layer is approaching the inviscid solution values.

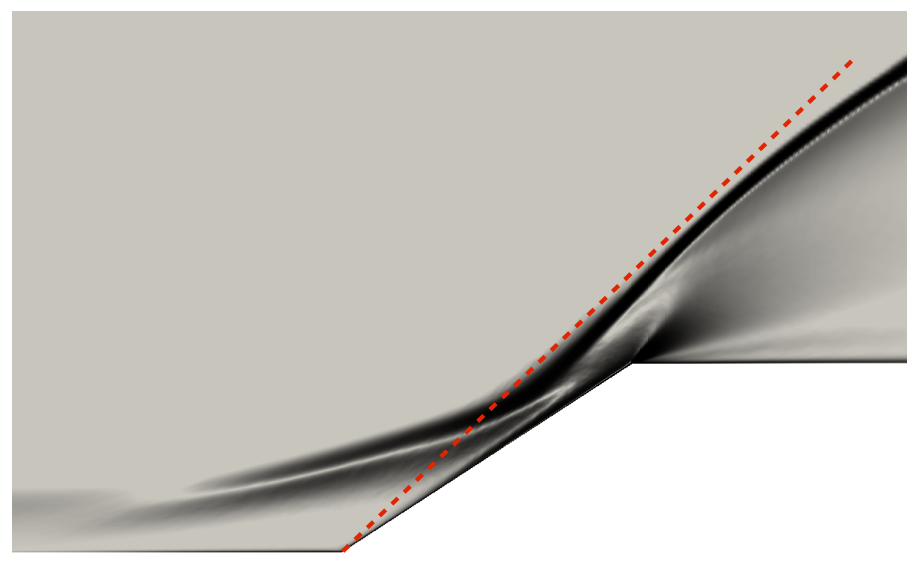

Figure 6: Numerical Schlieren of the averaged flow field in the simulation domain around the compression/expansion ramp. The red dashed line indicates the inviscid shock angle $\beta=43.9^{\circ}$.

In addition, a qualitative comparison of the instantaneous numerical Schlieren image from the iLES ramp flow is compared to the Schlieren image of the Mach 7.2 shock wave boundary layer interaction and separation bubble at the flare region of the FRESH FX-1 geometry produced by Wadhams et al. ${ }^{22}$ (Figure 8). The general form of the shock structure and the compression of the turbulence in the separated region agrees with the experimental image. 


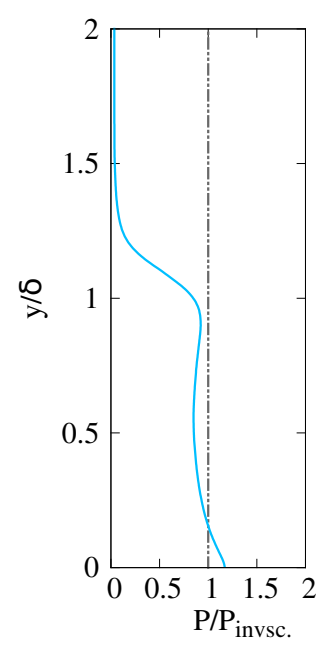

(a)

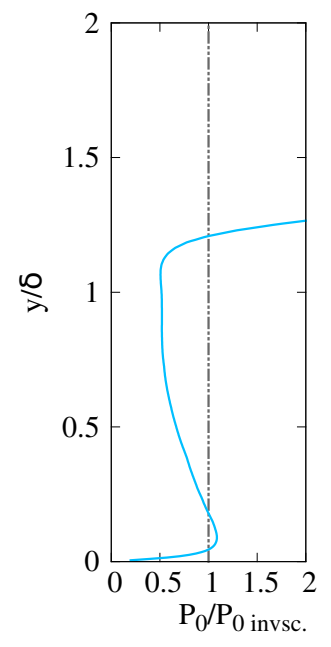

(b)

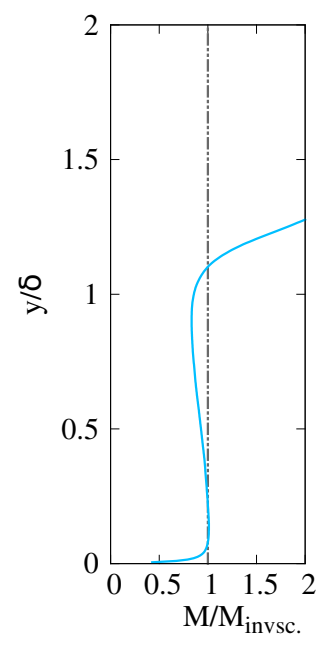

(c)

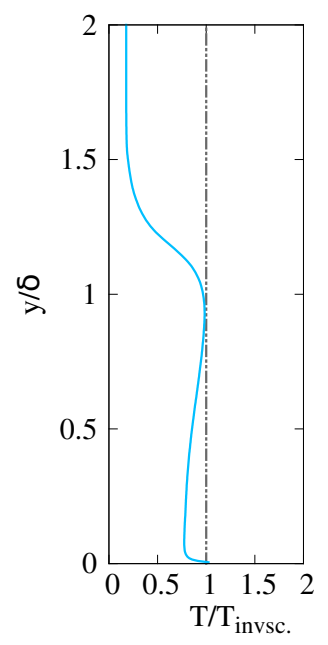

(d)

Figure 7: Temporal and spatial averaged flow field conditions compared to the inviscid oblique shock theory. The dashed line indicates the theoretical inviscid shock solution. The calculation point is on the ramp close to the expansion corner.

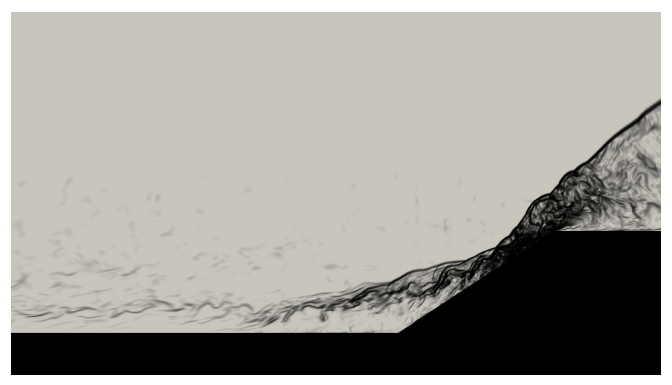

(a)

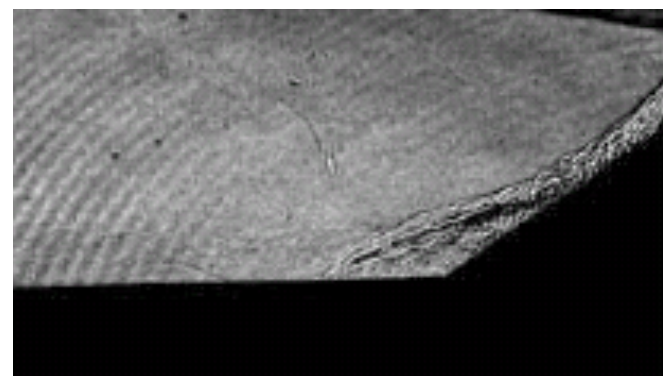

(b)

Figure 8: Qualitative comparison between iLES and experimental data. (a) Instantaneous numerical Schlieren $(N S=0.8 * \exp (-0.1|\nabla \rho|))$ of the ramp area. (b) Schlieren image of the Mach 7.2 SWBLI experiment by Wadhams et al. ${ }^{22}$ 


\section{High order statistics and physical insight}

The hypersonic SWBLI is further discussed below with reference to the Reynolds normal stress $\left(\tau_{u u}=\right.$ $\left.\rho \overline{u^{\prime} u^{\prime}} /\left(\rho_{w} u_{\tau}^{2}\right)\right)$ and high order statistics. The Reynolds normal stress has been normalized by the local wall density $\left(\rho_{w}\right)$, obtained from iLES, and the experimental friction velocity $u_{\tau}=57.3 \mathrm{~m} / \mathrm{s}^{7}$ A contour plot of $\tau_{u u}$ (Figure 9) shows high values of normal Reynolds stress $\tau_{u u}$, along the path of the separation surface and around the expansion corner; the predictions qualitatively agree with earlier experimental observations by Ueda and Hinze. ${ }^{23}$

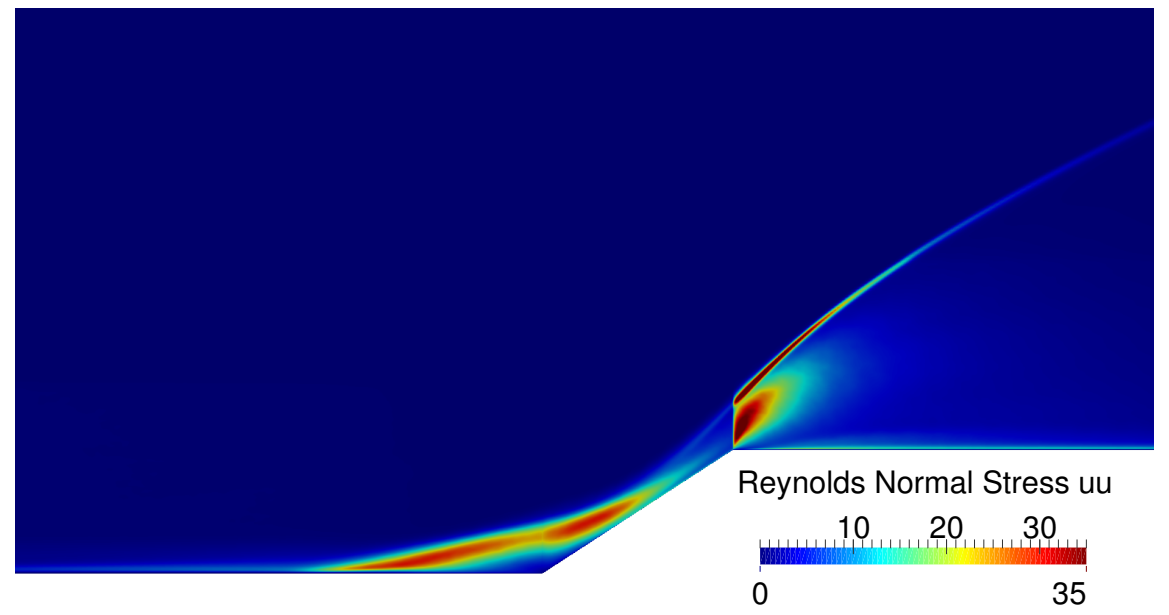

Figure 9: Contour plot of the Reynolds normal stress $\tau_{u u}$ around the compression/expansion ramp. The values have been temporally and spatially averaged (in span-wise direction).

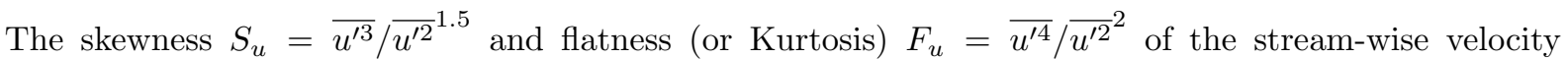
fluctuations are shown in Figures 10 and 11, respectively. The skewness or third moment is a measure of the asymmetry of the probability distribution of $u^{\prime}$, while flatness is the fourth moment that shows the frequency of events far from the mean value. ${ }^{24} \mathrm{~A}$ value of skewness around zero and a value of flatness around 3 indicate a Gaussian or symmetric probability distribution function. High values of flatness indicate a highly intermittent flow. It is expected that close to the wall and in the viscous sublayer the probability distribution of the stream-wise velocity fluctuations to be positively skewed, independently of the Reynolds number. ${ }^{25-27}$

Inside the separation bubble the velocity fluctuations are positively skewed over a greater distance above the wall due to the flow separation in this region (Figure 10). The flatness (Figure 11) is also above the Gaussian limit close the wall over the whole domain and to a greater extent inside the separation bubble due to the intermittent character of turbulence in the near wall region and inside the bubble. A close observation just above the near wall region upstream of the separation bubble shows that the minimum flatness value is observed at the same location as that for the zero-crossing skewness; this is similar to the observation made by Österlund and Johansson. ${ }^{28}$

Further away from the wall and in the logarithmic region of the boundary layer, turbulence returns to symmetry with $S_{u} \approx 0$ and $F_{u} \approx 3$. Close to the boundary layer edge negative values of skewness and positive values of flatness are observed. These suggest strong intermittency in this region dominated by small negative values of $u^{\prime}$, with infrequent but intense positive values. The foot of the lambda shock is creating a zone of highly negative skewness. This can be attributed to the motion/oscillation of the shock around its mean position which consequently creates very high values of velocity fluctuations. The expansion fan is characterized by the very high values of flatness. In combination with the small negative values of skewness in the same region this underlines the dominance of small negative values of $u^{\prime}$, while high positive values of $u^{\prime}$ are infrequent but extremely intense. In the free-stream, turbulence is symmetric, as expected, with $S_{u} \approx 0$ and $F_{u} \approx 3$. 


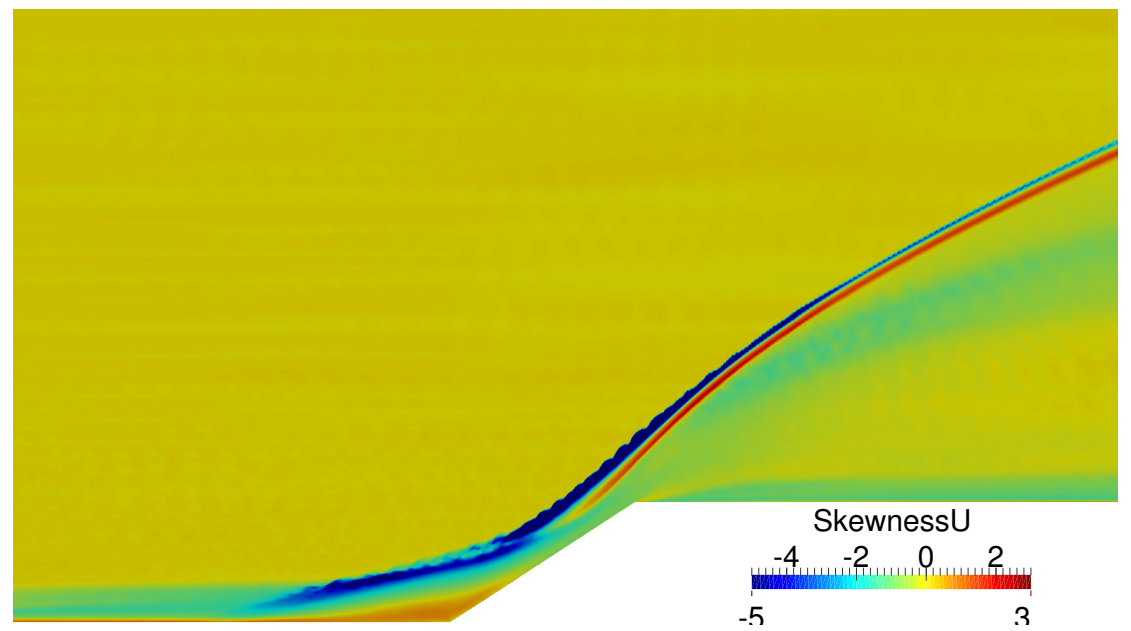

Figure 10: Contour plot of skewness $S_{u}$ around the compression/expansion ramp. The values have been temporally and spatially averaged (in span-wise direction).

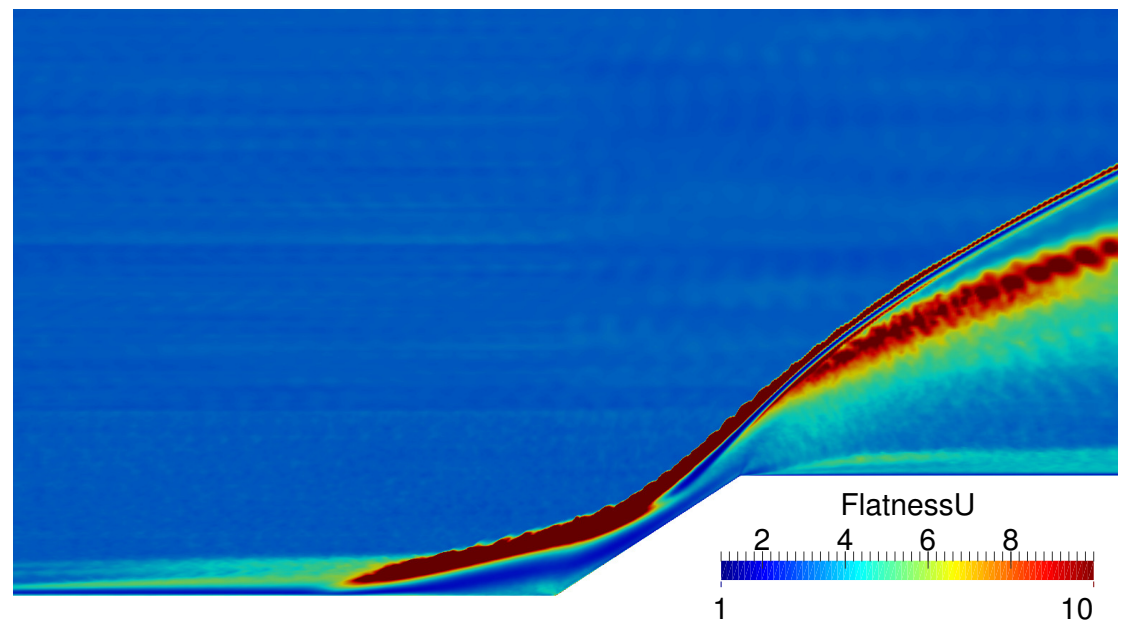

Figure 11: Contour plot of flatness $F_{u}$ around the compression/expansion ramp. The values have been temporally and spatially averaged (in span-wise direction).

\section{Conclusions}

An iLES study of hypersonic Mach 7.2 turbulent flow over a compression/expansion ramp has been presented. A grid convergence study was performed showing that a grid consisting of less than half the points of the finest grid provided very similar results. The iLES calculations were found to be in very good agreement with available experimental data and theoretical predictions. Key flow features have been identified and visualized through contour plots of the Reynolds normal stress $\tau_{u u}$ and high-order statistics, namely the skewness $S_{u}$ and flatness $F_{u}$ of the stream-wise velocity fluctuations $u^{\prime}$.

The results revealed that turbulence is highly asymmetric at the boundary layer edge, inside the separation bubble and around the shock regions. Due to flow separation the velocity fluctuations are positively skewed over a large distance above the wall. The intermittent character of turbulence in the near wall region and inside the bubble resulted in flatness values above the Gaussian limit. In agreement with previous publications the zero-crossing skewness value is observed to occur just above the near wall region at the same location as the minimum flatness value.

Strong intermittency was observed in the boundary layer edge, while the oscillation of the lambda shock created a zone of highly negative skewness. The analysis of the high-order statistics in the expansion fan region revealed a similar, though more intense behavior, as the one described for the boundary layer edge. As expected, in the free-stream turbulence is symmetric with $S_{u} \approx 0$ and $F_{u} \approx 3$. 


\section{Acknowledgments}

This work was sponsored by the Air Force Office of Scientific Research, Air Force Material Command, USAF, under grant number FA9550-14-1-0224. The U.S. Government is authorised to reproduce and distribute reprints for Governmental purpose notwithstanding any copyright notation thereon. The authors would like to thank S. M. Spottswood, Z. Riley and D. Garner for their support. The authors would also like to thank EPSRC for providing access to computational resources on the National HPC facility ARCHER (http://www.archer.ac.uk) through the UK Applied Aerodynamics Consortium Leadership Project "e529".

\section{References}

${ }^{1}$ Smits, A. J. and Dussauge, J. P., Turbulent Shear Layers in Supersonic Flow, Springer, 2006.

${ }^{2}$ Helm, C. M. and Martín, M. P., "New LES of a Hypersonic Shock/Turbulent Boundary Layer Interaction," 54th AIAA Aerospace Sciences Meeting, 2016, pp. 1-15.

${ }^{3}$ Helm, C. M. and Martin, P. M., "Görtler-like vortices in the LES Data of a Mach 7 STBLI," 55th AIAA Aerospace Sciences Meeting, 2017, pp. 1-13.

${ }^{4}$ Mikulla, V. and Horstmann, C. C., "Turbulence measurements in hypersonic shock-wave boundary layer interaction flows," AIAA Journal, Vol. 14, No. 5, 1976, pp. 568-575.

${ }^{5}$ Bookey, P., Wyckham, C., Smits, A. J., and Martín, M. P., "New experimental data of STBLI at DNS/LES accesible Reynolds numbers," 43rd AIAA Aerospace Sciences Meeting and Exhibit, No. AIAA 2005-309, 2005, pp. 1-18.

${ }^{6}$ Schreyer, A. M., Sahoo, D., and Smits, A. J., "Experimental investigations of a hypersonic shock turbulent boundary layer interaction," 49th AIAA Aerospace Sciences Meeting, No. AIAA 2011-481, 2011, pp. 1-12.

${ }^{7}$ Schreyer, A. M., Sahoo, D., and Smits, A. J., "Turbulence measurements with PIV in a hypersonic shock boundary layer interaction," 41st Fluid Dynamics Conference and Exhibit, No. AIAA 2011-3429, 2011, pp. 1-14.

${ }^{8}$ Drikakis, D., Hahn, M., Mosedale, A., and Thornber, B., "Large Eddy Simulation Using High Resolution and High Order Methods," Philos. Trans. Royal Soc. A, Vol. 367, 2009, pp. 2985-2997.

${ }^{9}$ Ritos, K., Kokkinakis, I. W., Drikakis, D., and Spottswood, S. M., "Implicit large eddy simulation of acoustic loading in supersonic turbulent boundary layers," Phys. Fluids, Vol. 29, No. 4, 2017, pp. 1-33.

${ }^{10}$ Ritos, K., Kokkinakis, I. W., and Drikakis, D., "Physical insight into the accuracy of iLES in turbulent boundary layers," Comput. Fluids, Vol. UNDER REVIEW, 2017.

${ }^{11}$ Toro, E. F., Riemann Solvers and Numerical Methods for Fluid Dynamics, Springer, 3rd ed., 2009.

${ }^{12}$ Balsara, D. S. and Shu, C. W., "Monotonicity preserving weighted essentially non-oscillatory schemes with increasingly high order of accuracy," J. Comput. Phys., Vol. 160, No. 2, 2000, pp. 405-452.

${ }^{13}$ Drikakis, D. and Rider, W., High-Resolution Methods for Incompressible and Low-Speed Flows, Springer, 2005.

${ }^{14}$ Blazek, J., Fluid Dynamics, Principles and Applications, Elsevier, 2015.

${ }^{15}$ Lund, T. S., Wu, X., and Squires, K. D., "Generation of turbulent inflow data for spatially-developing boundary layer simulations," J. Comput. Phys., Vol. 140, 1998, pp. 233-258.

${ }^{16}$ Klein, M., Sadiki, A., and Janicka, J., "A digital filter based generation of inflow data for spatially developing direct numerical simulation or large eddy simulations," J. Comput. Phys., Vol. 186, 2003, pp. 652-665.

${ }^{17}$ Touber, E. and Sandham, N. D., "Large-eddy simulation of low-frequency unsteadiness in a turbulent shock-induced separation bubble," Theor. Comp. Fluid Dyn., Vol. 23, 2009, pp. 79-107.

${ }^{18}$ Georgiadis, N. J., Rizzetta, D. P., and Fureby, C., "Large-eddy simulation: current capabilities, recommended practices, and future research." AIAA Journal, Vol. 48, No. 8, 2010, pp. 1172-1784.

${ }^{19}$ Choi, H. and Moin, P., "Grid-point requirements for large eddy simulation: Chapman's estimates revisited," Phys. Fluids, Vol. 24, 2012, pp. 011702.

${ }^{20}$ Poggie, J., Bisek, N. J., and Gosse, R., "Resolution effects in compressible, turbulent boundary layer simulations," Comput. Fluids, Vol. 120, 2015, pp. 57-69.

${ }^{21}$ Kolář, V., "Vortex identification: New requirements and limitations," Int. J. Heat Fluid Fl., Vol. 28, 2007 , pp. 638-652.

${ }^{22}$ Wadhams, T. P., Mundy, E., MacLean, M. G., and Holden, M. S., "Ground Test Studies of the HIFiRE-1 Transition Experiment Part 1: Experimental Results," J. Spacecr. Rockets, Vol. 45, No. 6, 2008, pp. 1134-1148.

${ }^{23}$ Ueda, H. and Hinze, J. O., "Fine-structure turbulence in the wall region of a turbulent boundary layer," J. Fluid Mech., Vol. 67, 1975, pp. 125-143.

${ }^{24}$ Erm, L. P., Low Reynolds-number turbulent boundary layers, Ph.D. thesis, University of Melbourne, 1988.

${ }^{25}$ Kim, J., Moin, P., and Moser, R., "Turbulence statistics in fully developed channel flow at low Reynolds number," $J$. Fluid Mech., Vol. 177, 1987, pp. 133-166.

${ }^{26}$ Jiménez, J. and Pinelli, A., "The autonomous cycle of near-wall turbulence," J. Fluid Mech., Vol. 389, 1999 , pp. 335-359.

${ }^{27}$ Pirozzoli, S. and Bernardini, M., "Turbulence in supersonic boundary layers at moderate Reynolds number," J. Fluid Mech., Vol. 688, 2011, pp. 120-168.

${ }^{28}$ Österlund, J. M. and Johansson, A. V., "Turbulence statistics of zero pressure-gradient turbulent boundary layers," Tech. rep., Sweden: KTH Mechanics, 1999. 